\title{
On The Formal-Logical Analysis of the Foundations of Mathematics Applied to Problems in Physics
}

\author{
Temur Z. Kalanov \\ Home of Physical Problems, \\ Pisatelskaya 6a, 100200 Tashkent, Uzbekistan. \\ Email: tzk_uz [AT] yahoo.com
}

\begin{abstract}
1. As is well known, mathematical physics is the fundamental science of "the application of mathematics to problems in physics and the development of mathematical methods suitable for such applications and for the formulation of physical theories" (The Journal of Mathematical Physics). Mathematical physics arose from the needs of science and practice and has a long history of development. The important significance of this field of science is determined by the contribution of prominent scientists of past and present times. However, it does not mean that the problem of validity of the achievements of mathematical physics is now completely solved, or that the foundations of mathematics and physics are not in need of analysis within the framework of the correct methodological basis: the unity of formal logic and of rational dialectics. In my opinion, standard mathematical and physical theories can not be considered as scientific truth if there is no formal-logical and dialectical substantiation of it in science.
\end{abstract}

2. The correct methodological basis represents theoretical generalization of practice and, therefore, is the criterion of truth of theories. In this connection, the following questions arise: How can we apply formal-logical and dialectical laws to the analysis of the foundations of the special sciences? Do mathematical and physical theories obey the formal-logical and dialectical laws? In my opinion, the formal-logical law of identity and the dialectical-materialistic law of measure (i.e., the law of interrelation, of interdependence, of inter-conditionality of qualitative and quantitative aspects (determinacy) of a material object) can be used in mathematics and physics in the following wording: the left-hand side and the right-hand side of the mathematical (i.e., the quantitative) relationship must have identical qualitative determinacy. As first shown in my published works, the use of this statement leads to the following conclusion: the foundations of theoretical physics and of mathematics are not free from objections because the standard theories do not satisfy the criterion of truth.

3. In my opinion, a thorough understanding of the foundations of mathematics is impossible without a critical analysis of the concept of mathematical quantity - central concept of mathematics. A critical analysis of the concept of mathematical quantity leads to the following conclusion:

The concept of "mathematical quantity" is the result of the following mental operations: (i) abstraction of the "quantitative determinacy of physical quantity" from the "physical quantity" at that the "quantitative determinacy of physical quantity" is an independent object of thought; (ii) abstraction of the "amount (i.e., abstract number)" from the "quantitative determinacy of physical quantity" at that the "amount (i.e., abstract number)" is an independent object of thought. In this case, unnamed, abstract numbers are the only sign of the "mathematical quantity". This sign is not an essential sign of the material objects. Therefore, the content of the concept of "mathematical quantity" is zero, and the volume of this concept is infinitely large.

Thus, the concept of mathematical quantity is meaningless, erroneous, and inadmissible concept in science because it represents the following formal-logical and dialectical-materialistic error: negation of the existence of the essential sign of the concept (i.e., negation the existence of the essence of the concept) and negation of the existence of measure of material object.

4. Mathematical theories can be applied to problems in physics if and only if the mathematical relationships are interpreted geometrically or physically. The interpretation is that the mathematical quantities in the standard relationship $y=f(x)$ are associated with geometric (metric) or physical quantities $x_{M}$ and $y_{M}$ characterizing the material object $\boldsymbol{M}$. Interpretation operation represents replacement $x \rightarrow x_{M}, y \rightarrow y_{M}$ in the relationship $y=f(x)$. In fact, the interpretation is expressed by the identities $x=x_{M}, y=y_{M}$. In this case, the relationship between the physical quantities has the form $y_{M}=f\left(x_{M}\right)$ and can be tested in practice. This relationship means that the interpretation leads to the restoration of the measure of the material object (i.e., the restoration of the unity of qualitative and quantitative determinacy of the material object). Pure mathematics (i.e., mathematics without restored measure) is, according to Einstein, useless science. Therefore, the interpretation is a criterion of truth in pure mathematics. 
5. In my published works, analysis of the foundations of mathematics applied to problems in physics was proposed. It was first shown within the correct methodological basis that the foundations of differential and integral calculus, the foundations of vector calculus, the Pythagorean theorem, the foundations of trigonometry, and the foundations of the theory of negative numbers are not free from objection because these standard results contain the formal-logical and dialectical-materialistic errors. Removing these errors leads to the abolition of many standard theories. And the abolition of the standard theories turns science into helpless and barren knowledge.

In my opinion, the errors are an inevitable consequence of the inductive method of cognition. 